
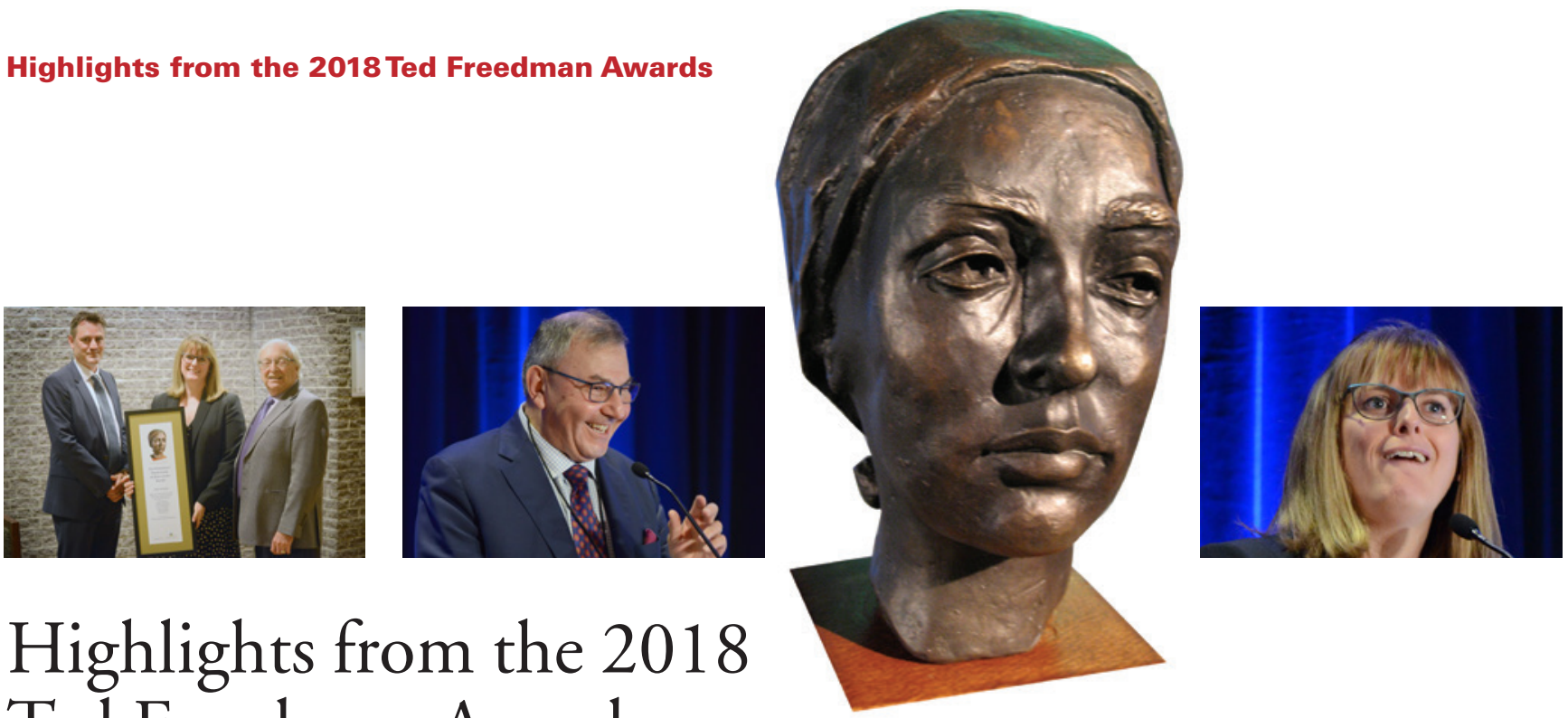

\title{
Highlights from the 2018 Ted Freedman Awards
}

In the year 2000, Ted Freedman, former president and CEO of Toronto Mount Sinai Hospital and a founding member of the Healthcare Quarterly editorial advisory board, was the surprised honoree of an annual award launched by the journal in cooperation with Agilent Technologies. The award recognizes those people who inspire, advocate and enable education in healthcare. The winners are honoured with a self-portrait in bronze by award-winning sculptor Amy Switzer of Barrie, Ontario. Ms. Switzer was recovering from brain surgery when she created the bust (see above).

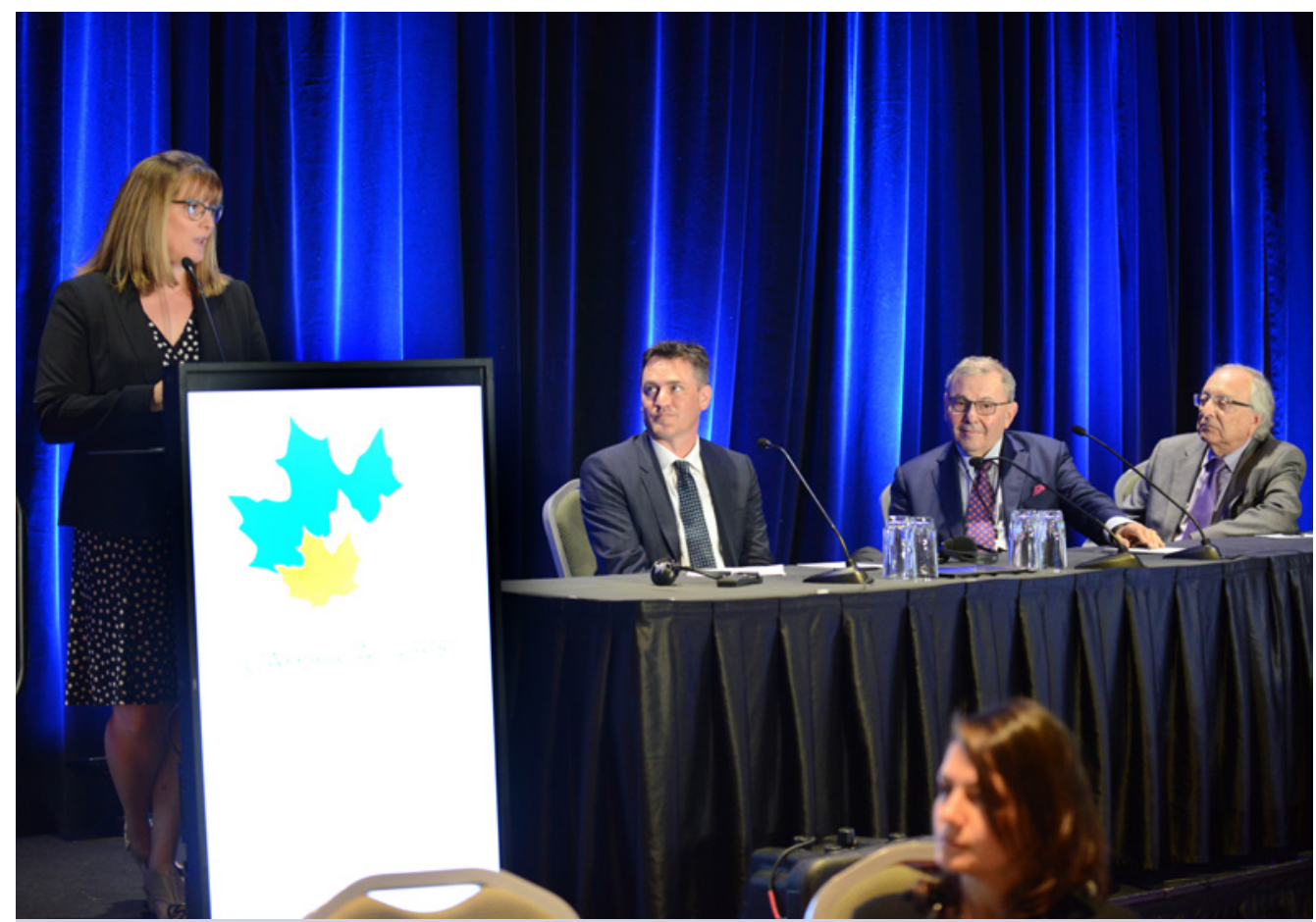

Raquel Meyer, Baycrest, accepting the 2018 Ted Freedman Award with Matthew Hart, Frank Markel and Ted Freedman.
THANK YOU TO THIS YEAR'S ESTEEMED PANEL OF JUDGES:

Ted Freedman

Matthew Anderson, President \& CEO of Lakeridge Health

Rhonda Cockerill, Acting Director of the Institute of Health Policy, Management and Evaluation at the University of Toronto

Frank Markel,

Executive Director, Canadian Association for Health Services and Policy Research

Rebecca Hart,

Publisher,

Longwoods Publishing 


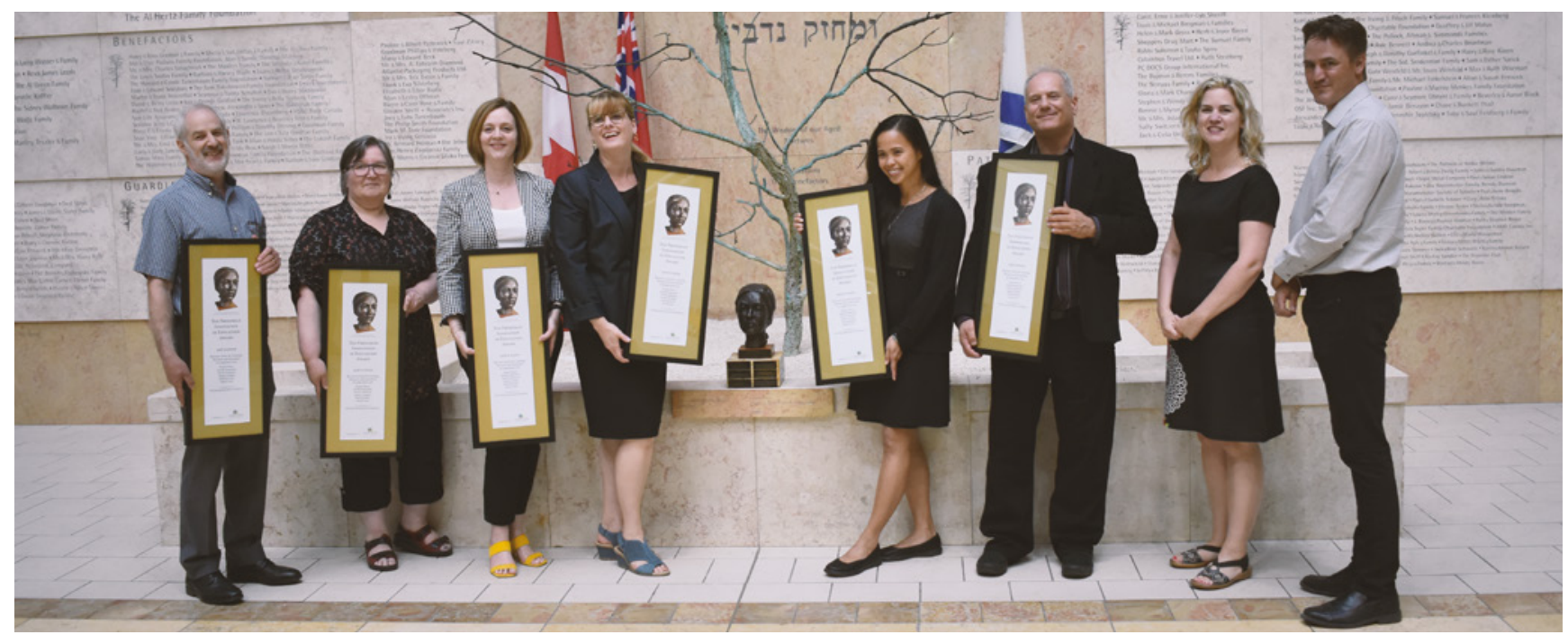

SOS Educational App for Healthcare:

\section{Improved Clinical Reasoning through Simulation and Game-Based Learning}

On our journey to transform gerontological education and practice in long-term care, we were seeking solutions to:

- provide post-workshop skill rehearsal with feedback given that learning is not a one-time event;

- meet the demand for microlearning, in the context of scarce resources;

- demonstrate improved care competence, a key precursor to quality and safety outcomes;

- re-engage teams in cultures of learning based on leading educational practices; and

- enhance the profile of long-term care because aging care is where innovation is happening!

As a result, we developed the SOS Educational App for Healthcare - an educational innovation that combines simulation and game-based learning to enhance knowledge application and clinical reasoning. Given the significant risk of patient morbidity and mortality in healthcare, analytical and reflective clinical reasoning is essential to reduce error rates and adverse events. Clinical reasoning, the process by which clinicians recognize, assess, reflect on, intervene in, and evaluate patient situations, requires self-awareness and the use of metacognitive processes in the application of a decision-making framework. Clinicians are also required to integrate evidence-based guidelines and research into care in order to achieve quality standards.

The SOS Educational App for Healthcare achieves these objectives while modelling reality and offering depth of challenge via dynamic decision-making points and real-world options. Through a series of patient simulations, players are challenged to recognize and prioritize symptoms, identify patterns, make efficient clinical decisions and rehearse communication. A gamified virtual patient system links together
38 cases for geriatric medical conditions associated with preventable hospitalizations. Based on real patient situations, the case simulations integrate evidence from 27 clinical guidelines, 25 evidence-based syntheses and 18 research publications. A Sensory Observation System (SOS) ensures systematic clinical assessment. SBAR (Situation - Background Assessment - Recommendation) is used to structure each case and rehearse clinical communication.

The virtual patient system is grounded within a serious educational game. Game mechanics include action point starvation, randomization, virtual earnings, time constraints, difficulty levels, player levels and power-ups to produce a deeper game. These types of gamified mechanics have been shown to deactivate default mode processing in the brain and stimulate the ventral striatum, the main learning reward centre. The app also remedies learners' needs for rehearsal, promotes active and self-paced learning and consolidates skills through repeated case presentations using randomized stems and answer sets. Self-monitoring and self-assessment are enabled through frequent and instant performance feedback (reflective prompts, player statistics, badges and leaderboards).

In addition, the app utilizes problem-based learning theory on pattern recognition, in conjunction with the neuroscience of decision making. Gamification of simulation not only increases engagement but, more importantly, improves decision making by forcing players to slow down to select the correct decision path and to reflect on clinical reasoning. The app's game mechanics require players to select the correct course of clinical action, focusing their attention on the unique details of the virtual patient encounter. As an example, the game mechanic of action point starvation limits the number of actions a player can make before a game ends. In the app, this forces players to focus assessment choices based on the patient situation and medical history, thus fostering reflection and efficiency in the 
clinical decision-making process. Rather than applying knowledge sequentially to a known medical condition, learners must consider multiple conditions under real-world conditions and integrate evidence to solve a variety of cases.

The app also tackles four pervasive pedagogical challenges: passive learning, which leads to lower academic performance than active learning; a focus on knowledge acquisition without sufficient depth of challenge to evaluate application and performance change; the desire for more rehearsal with immediate feedback to practise and self-monitor performance and the use of simulated patients and case-based discussions which are resource-intensive with limited scalability. Analytics enable educators and employers to monitor performance and respond 'just-in-time' to learning gaps. Any type of self-authored case content can be uploaded - the app platform can be readily adapted for other clinical populations and any number of healthcare disciplines or roles. The SOS Educational App can supplement blended learning and enables accessible and scalable mobile learning to an unlimited number of learners anywhere, anytime, on any device.

Pilot studies with nursing students showed increases in self-reported knowledge and confidence after three weeks of gameplay and satisfaction with the app. Statistically significant improvements in learning curves were also associated with gameplay - the more learners played, the more cases they solved! That is, case-solved rates generally increased as more cases of the same type were played. Thus, improvements in knowledge application and clinical reasoning relative to real-world patient scenarios were achieved through the app.

For more information, visit www.clri-ltc.ca or connect with us to explore the use of the SOS Educational App for Healthcare with your learners and clinical population.

\section{Ted Freedman Awards - A Selection of Submissions}

\begin{abstract}
“By Us For Us" Guides
When Brenda Hounam was diagnosed with Alzheimer's disease at the age of 53, she realized that very little information was available for people living with dementia. It was then that she decided to take things into her own hands. Brenda gathered a small group of her peers, who were also living with memory loss, and they decided to write a guide providing suggestions on ways to keep your brain active. This guide, "Memory Workout," became the first in a series of short, practical resources known as the By Us For Us(C) (BUFU) guides. With the support of the Murray Alzheimer Research and Education Program (MAREP) at the University of Waterloo in Ontario, one guide has grown into four unique series with a total of 13 individual publications, all researched and developed by a group of talented and passionate people living with dementia. The guides are designed to equip people living with dementia with the necessary tools to live well with dementia. What makes these guides particularly useful is that they are created BY people living with dementia FOR people living with dementia, so the information is relevant, useful and, most importantly, based on lived experience.
\end{abstract}

The BUFU project has been tremendously successful and has had a huge impact on people living with dementia, family members and professionals all over the world. Over 200,000 copies of the printed BUFU guides have been distributed globally and are available in three languages: English, French and Spanish.

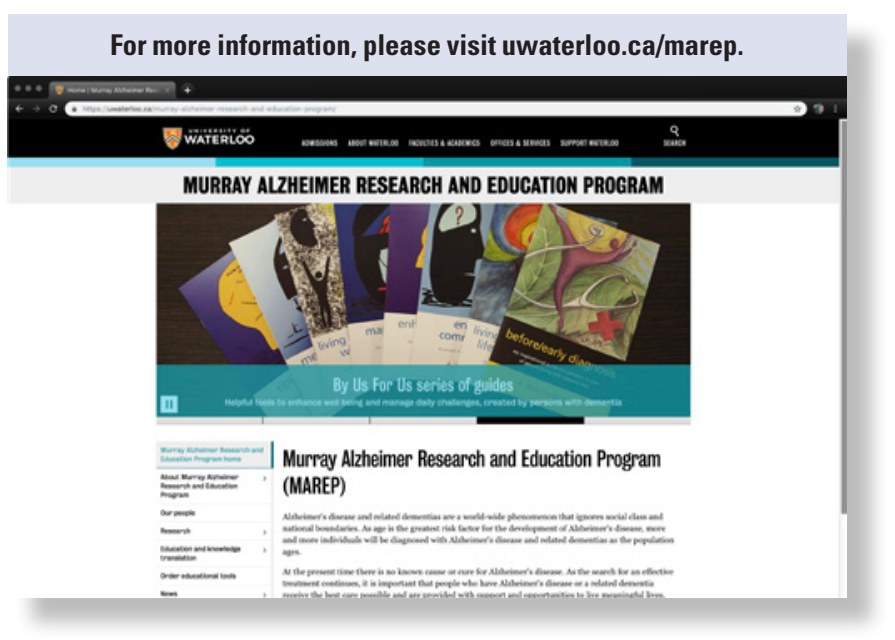

\section{Accelerated Education Program (AEP)}

The Accelerated Education Program (AEP) at the Princess Margaret Cancer Centre addresses the practical learning needs of radiation medicine healthcare professionals around the world. Since its development in 2005, AEP has enabled successful dissemination of cutting-edge research in radiation medicine and practical knowledge to professionals, patients and the public using tele-education, webcasting and novel simulation tools. AEP helps practitioners integrate emerging technologies into the clinical arena, use research findings to inform and improve practice and promote innovative 
inter professional workplace models to accelerate the adoption of clinical innovations, such as image-guided radiation therapy (IGRT), to improve the quality and safety of radiation medicine and, ultimately, patient outcomes.

AEP's educational offerings include in-depth structured two- to three-day courses in IGRT, intensity modulated RT (IMRT), quality and safety in RT (QSRT) and accelerator technology (ATec) - the only course in the world tailored to medical physicists that offers an immersive environment to learn about linear accelerators. In addition, AEP has developed seven site-specific IGRT courses, including Lung Stereotactic Body RT (SBRT), Liver SBRT, Head \& Neck IM/IGRT, Brain Tumor, Paraspinal, Oligometastases and Soft Tissue Sarcoma. These interactive and interdisciplinary courses have attracted over 1,300 radiation medicine professionals and trainees from 32 countries around the world, spanning all six continents. The impact of our award-winning program has been widely recognized nationally and internationally through numerous successful collaborations. Collectively, AEP offers unique opportunities to acquire the knowledge, skills and strategies necessary to implement state-of-the-art radiotherapy practices worldwide.

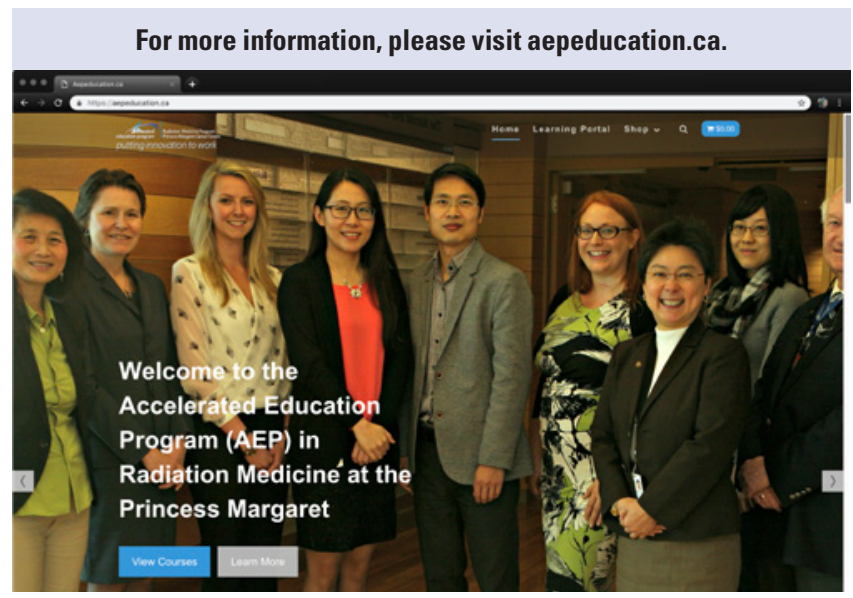

\section{Canadian Federation of Medical Students, (CFMS) National Day of Action on Indigenous Mental Wellness}

In February 2018, the Canadian Federation of Medical Students (CFMS) held a National Day of Action on Indigenous Mental Wellness. This innovation is a demonstration of the collective advocacy efforts of medical students to lend our voices as allies to an issue of dire importance, an exercise of our abilities to mobilize and an experience in teaching one another on a health inequity issue while gaining practical advocacy skills. The process involved an extensive consultations process with Indigenous leaders and health experts. A qualitative analysis was completed to review the findings to help identify policy issues, which are as follows:

1. Adopt the First Nations Mental Wellness Continuum Framework as a framework to address First Nations peoples' mental health and suicide.

a. Adopt the National Inuit Suicide Prevention Strategy as a framework to address Inuit peoples' mental health and suicide.

b. Undertake a comprehensive review of the current distribution of funding through the National Aboriginal Youth Suicide Prevention Strategy (NAYSPS) in collaboration with Indigenous communities, to ensure that every Indigenous community receives funding that is both sustainable and provided in accordance with need.
2. Direct Health Canada and Indigenous Services Canada to re-evaluate what programs and services are funded under the Non-Insured Health Benefits Program (NIHB) and

a. Increase funding for preventive and land-based mental wellness programs that create opportunities within the community.

b. Support and expand the list of approved service providers to include Indigenous traditional knowledge keepers.

Following the Day of Action, we are continuing to mobilize and ensure that our efforts are longitudinal, accountable and sustainable, including holding an AFN/ CFMS Joint Virtual Forum on Indigenous Mental Wellness.

For more information and the summary report, visit cfms.org/what-we-do/advocacy/day-of-action.html.

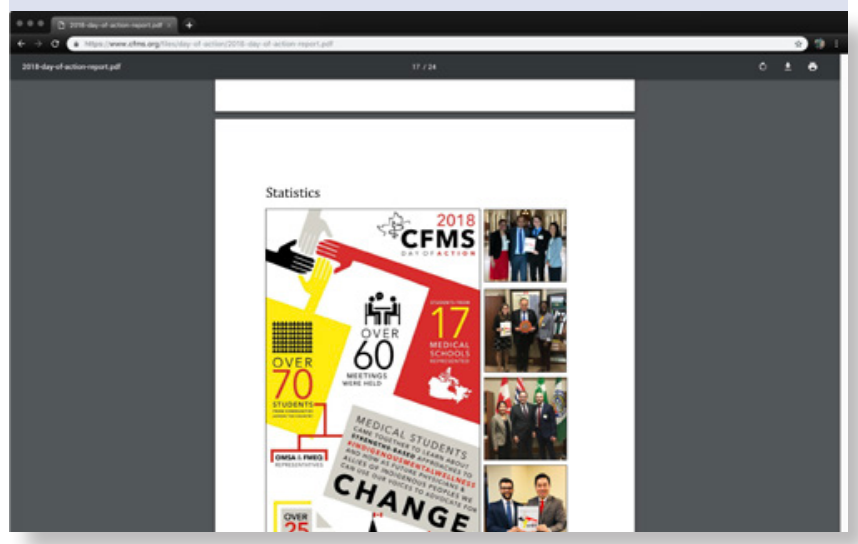

